\title{
Effects of the relative humidity and water droplet on adhesion of a bio-inspired nano-film
}

\author{
Zhilong Peng, Shaohua Chen* \\ LNM, Institute of Mechanics, Chinese Academy of Sciences, Beijing 100190, China
}

\section{A R T I C L E I N F O}

\section{Article history:}

Received 15 February 2011

Received in revised form 6 July 2011

Accepted 8 August 2011

Available online 16 August 2011

\section{Keywords:}

Gecko

Nano-film

Adhesion

Relative humidity

Water droplet

\begin{abstract}
A B S T R A C T
Inspired by geckos' adhesion, the effect of water membrane forming due to the environmental humidity, on the adhesion between a bio-inspired nano-film and a substrate is investigated first. The disjoining pressure is considered, which results in an enhancing adhesion between the nano-film and substrate. When the thickness of water membrane increases, water droplets will form and a repulsive capillary force between the nano-film and substrate is produced. The total adhesion force decreases with an increasing volume of water droplets. The two opposite results in the two different models are consistent well with two seemingly inconsistent experimental observations by Huber et al. (2005) [4] and Sun et al. (2005) [5], respectively, and may be significant for the development of artificial biomimetic attachment systems.
\end{abstract}

(c) 2011 Elsevier B.V. All rights reserved.

\section{Introduction}

Understanding the adhesion mechanisms of biological systems not only provides insights into biological attachment systems but also may yield general principles employed in nature as inspiration and guidance for development of biomimetic adhesive devices. Several creatures including insects, spiders and geckos have a unique ability to climb and detach from ceilings and vertical walls using their attachment systems. Experiments have found that there are small hairs covering the surfaces of the pads of their feet, called setae. Each seta branches into hundreds of spatulae. Measurement of the adhesion force of a single seta was carried out by Autumn et al. [1]. Atomic force microscopy was used to determine the pulloff force of even a single spatula [2]. The majority of experimental results point towards van der Waals force as a dominant mechanism of adhesion [1,3]. However, the measurements of the adhesion force exerted by a single gecko spatula for various atmospheric conditions suggest that capillary force can be a contributing adhesive factor [4,5]. Humidity contributes significantly to gecko adhesion and the pull-off force will increases with increasing relative humidity [4,5]. Several theoretical models have been established to try to explain this interesting phenomenon [4-6]. The effect of relative humidity is often expressed by a liquid bridge between a sphere and a substrate. However, the spatulae on seta's end are not simple pillars or spheres, which look like nano-films and might

\footnotetext{
* Corresponding author. Tel.: +86 10 82543960; fax: +86 1082543977.

E-mail address: chenshaohua72@hotmail.com (S. Chen).
}

be expected to be most important in determining the adhesion of geckos. Furthermore, Orr et al. [7] indicated that ordinary vapor could not condense to form a liquid bridge between hydrophobic or hydrophobic and hydrophilic surfaces spontaneously. Recently, from the material point of view, Prowse et al. [8] and Puthoff et al. [9] reported that relative humidity can change materials properties and an increase in relative humidity softened setae and increased viscoelasitc damping, which increases adhesion. Chen and Gao [10] numerically analyzed the reduction in material stiffness induced by moisture, which leads to substantial increases in the pull-off force of the spatula pad. But the influence of the relative humidity on interfacial adhesion mechanism has not reached a unified perspective. Huber et al. [4] found that water membrane similar to a monolayer of water forms between two adjacent surfaces due to the environmental humidity. At a humidity of $88 \%$, the thickness of the water-film is about $0.2 \mathrm{~nm}$, which corresponds roughly to one additional monolayer of water. In fact, DelRio et al. [11] studied the capillary adhesion for micromachined contacting surfaces and also found that the adsorbed water layer thickness is only about one or two monolayers at more than $70 \%$ relative humidity. The adsorbed water membrane is so thin that the attractive force between liquid molecules and substrate is very strong. Thus the disjoining pressure [12] between liquid layer and substrate must be considered. In fact, the attractive disjoining pressure between a polymeric liquid film and a substrate was proved by Mate and Novotny [13] experimentally. It was found that the disjoining pressure indicated strong attractive interaction between the polymer molecules and the solid surface when the thickness of liquid film was very thin, and decreased with increasing film thickness in a manner consistent 


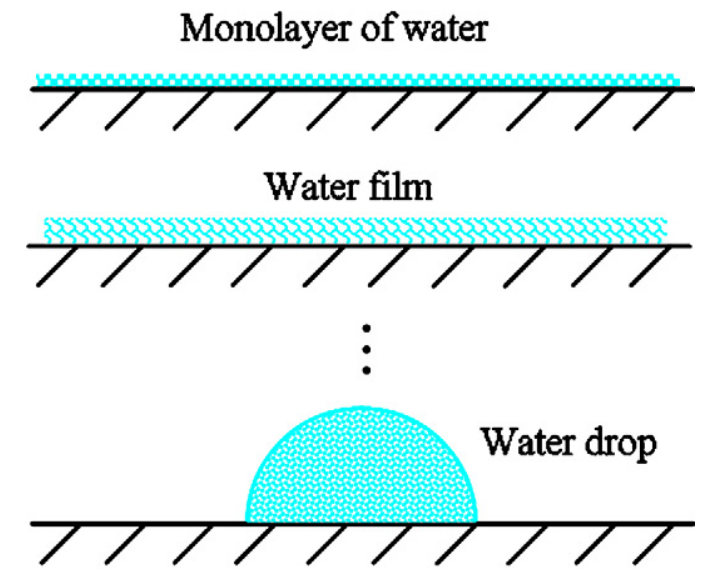

Fig. 1. Schematic of the transformation from a monolayer of water to a water droplet on a substrate.

with a strong van der Waals interaction between liquid molecules and solid surface. The flat monolayer liquid conformation on substrate indicated that liquid molecules gained more energy from the strong attractive interaction than they would gain in entropy by adopting bulklike conformation. In other words, when the liquid film becomes thick enough, the disjoining pressure becomes so weak that entropy would cause the liquid molecules to adopt a more bulklike conformation [13], such as liquid droplets, as shown in Fig. 1.

In order to explain the experimental observation, Huber et al. [4] assumed that an areal fraction $f$ of geckos' spatula is in direct contact with the substrate and another fraction $f$ of the spatula contacts with the substrate through a water membrane. However, in their work, the ratio of $f$ and $f$ was assumed to be a constant and independent of the varying relative humidity, which is not true.

In this paper, the spatula is represented as a finite scale nanofilm. The adhesion between the nano-film and a rigid substrate with a partial coverage by a monolayer of water is investigated first. Due to the very thin thickness of the adsorbed monolayer of water between the nano-film and substrate, the change of effective short-range interaction should be considered. The disjoining pressure induced by the adsorbed water layer may contribute to the total adhesion force. When the relative humidity exceeds $90 \%$, a "real" capillary condensation would be expected to occur. Then, a model of a nano-film contacting a substrate with an intermediate water bridge will be considered. In this case, the deformation of nano-film induced by the liquid surface tension is considered and the capillary force, van der Waals force, and total adhesion one are calculated as a function of the volume of liquid droplet.

\section{Theoretical model considering relative humidity and water droplet}

\subsection{The effect of relative humidity}

Consider an adhesive model with a monolayer of water molecule between a nano-film and a substrate first. As we know that Mate and Novotny [13] ever studied the molecular conformation and disjoining pressure of polymeric liquid films. It was found that, for a monolayer of liquid film, the disjoining pressure indicated strong attractive interaction between the liquid molecules and the solid surface, so that liquid molecules lied flatly on the substrate, and the disjoining pressure would decrease with an increasing film thickness [13]. Furthermore, Huber et al. [4] confirmed experimentally that only a monolayer of water was adsorbed on substrate when relative humidity was low. Thus disjoining pressure should

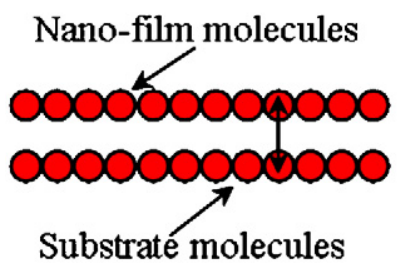

(a)

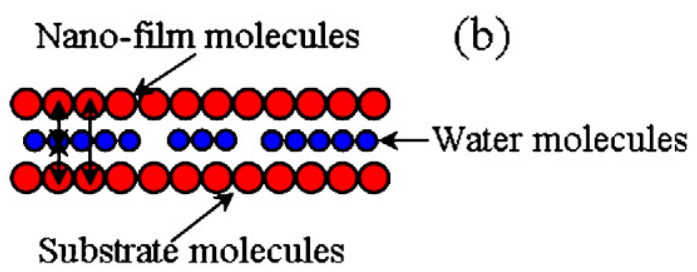

Fig. 2. Schematic of the contribution of the disjoining pressure to the adhesion force between a nano-film and a substrate. (a) A nano-film is in direct contact with a substrate. (b) A nano-film contacts with a substrate through a monolayer of water molecules.

be considered when such a thin water film is adsorbed on surfaces. Disjoining pressure was first introduced by Derjaguin in 1936. It is one of the fundamental physical parameters determining the properties of liquid films. For a liquid film on a solid surface, the disjoining pressure representing the interaction energy per unit volume between liquid layer and solid substrate has three independent components, $\Pi=\Pi_{w}+\Pi_{e}+\Pi_{s}$, where $\Pi_{w}$ is the pressure from the van der Waals force acting between the film and the substrate, $\Pi_{e}$ the ionic electrostatic component, and $\Pi_{s}$ the structural component resulting from the molecules having a different structure or conformation in the film compared to the bulk liquid. The main component is van der Waals force acting between the liquid layer and the substrate, which can be expressed as $\Pi=A / 6 \pi h^{3}$ (where $A$ is the Hamaker constant between liquid molecule and solid substrate, and $h$ is the thickness of liquid layer) $[13,14]$. The van der Waals disjoining pressure was widely used to study the interaction between surfaces with water interlayer [15-19]. When a monolayer of water molecules adsorb on substrate, the contribution of disjoining pressure can be divided into two parts as shown in Fig. 2. When the nano-film directly contacts a substrate, the adhesion force of the nano-film on the substrate is only the molecular forces between the nano-film and substrate (Fig. 2a). While the adhesion force consists of not only the molecular force between nano-film and substrate but also the contribution from the interactions between the water molecules and nano-film in the case of a nano-film contacting with a substrate through a monolayer of water molecules (Fig. 2b). In this case, the property of monolayer of water film is similar to solid molecules, which will enhance the interactions.

According to the experimental observation [4], at a relative humidity of $88 \%$, the water layer thickness on substrate increases by about $0.2 \mathrm{~nm}$, which corresponds roughly to one additional monolayer of water. Assuming that the amount of liquid is in thermal equilibrium with the vapor phase, the relative water coverage $\rho$ expressed by relative humidity is given by Langmuir's adsorption isotherm [4]:

$\rho=\frac{R H}{R H+\exp \left(-E_{a} / \kappa_{B} T\right)} \approx R H \times \exp \left(\frac{E_{a}}{\kappa_{B} T}\right) \approx 1.22 R H$

where $R H$ is the relative humidity, and $E_{a}$ is the adsorption energy that is typically much less than the thermal energy $\kappa_{B} T$ at room temperature. Here, one should be noted that the water coverage $\rho$ is a function of the relative humidity and not a constant as that used 


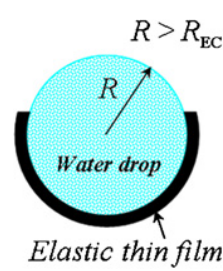

Fig. 3. Schematic of a water droplet interacting with a soft thin film. $R_{\mathrm{EC}}$ is an elastocapillary length [20]. When the radius of $R$ is not less than $R_{\mathrm{EC}}$, complete wrapping happens.

in Huber et al. [4]. Clearly, Eq. (1) does not apply when $R H$ is larger than $90 \%$ because the water coverage $\rho$ is larger than one at this time. The disjoining pressure describing the interaction between liquid molecules and solid substrate can be written as

$F_{\mathrm{SL}}=\frac{A}{6 \pi h^{3}} \rho b_{1} b_{2}$

where $b_{1}, b_{2}$ are the length and width of elastic thin film, respectively. Due to the very thin thickness of the water layer, the two solid surfaces lie in the effective interacting distance of van der Waals force. The effective Hamaker constant describing the interaction between the two solid surfaces can be expressed as,

$A_{\text {eff }}=(1-\rho) A_{\text {dry }}+\rho A_{\text {wet }}$

where $A_{\text {wet }}$ is the Hamaker constant with a monolayer of water, while $A_{\mathrm{dry}}$ is the one without water layer between the solid surfaces. Thus the van der Waals force between the two solid surfaces is

$F_{\mathrm{SS}}=\frac{A_{\mathrm{eff}}}{6 \pi D_{0}^{3}} b_{1} b_{2}$

where $D_{0}$ is the surface separation, which equals to the thickness of water layer $h$. The total adhesion force is then given by

$F_{\text {total }}=F_{\mathrm{SS}}+F_{\mathrm{SL}}=\left[\frac{(1-\rho) A_{\mathrm{dry}}}{6 \pi D_{0}^{3}}+\frac{\rho A_{\mathrm{wet}}}{6 \pi D_{0}^{3}}+\frac{\rho A}{6 \pi h^{3}}\right] b_{1} b_{2}$

In Eq. (5), van der Waals force between the nano-film and substrate is weakened by the intermediate water layer, while the strong attractive disjoining pressure between water molecules and solid surface compensates the reduced interaction and may enhance the adhesion of two solid surfaces.

\subsection{The effect of water droplet}

As the water layer becomes thicker, the disjoining pressure between liquid layer and substrate decreases. Water molecules gain less energy from the attractive interaction of substrate than they would gain in entropy by adopting a conformation that would extend them significantly above the surface. Thus, entropy causes molecules to adopt a more bulklike conformation [13], such as liquid droplet as shown in Fig. 1.

Assuming the radius of the liquid droplet is much smaller than the capillary length, $L_{c}=\left(\gamma / \rho_{0} g\right)^{1 / 2}$ (where $\gamma$ and $\rho_{0}$ are the liquid surface tension and density), thus the gravity of liquid is negligible, which results in a spherical droplet. Since the bending rigidity of the nano-film is very small, deformation will happen to match the droplet shape under the surface tension of liquid droplet, i.e., capillary wrapping as shown in Fig. 3. Considering a thin film with length $L$ in contact with a droplet of radius $R$. If the film is sufficiently compliant, it will fold around the droplet. The gained surface energy, $\gamma(1+\cos \theta) L$ (per unit width, $\gamma$ being the surface tension and $\theta$ the contact angle), must be balance with the bending energy, $D L / 2 R^{2}$ where $D=E t^{3} / 12\left(1-v^{2}\right)$ is the bending stiffness ( $E$ is the Young's modulus, $v$ the Poisson's ratio and $t$ is the film thickness) [20]. The energy balance gives $R_{\mathrm{EC}}=\sqrt{D / 2 \gamma(1+\cos \theta)}$, which is referred as an elasto-capillary length [21]. $R_{\mathrm{EC}}$ corresponds to the typical radius of curvature that capillary forces may generate on a solid with a given bending rigidity. Therefore, the state of a thin film wrapping a liquid droplet completely is stable if the radius $R$ of liquid droplet is larger than $R_{\mathrm{EC}}$ (Fig. 4a). The capillary force, under this circumstance, can be divided into two components: the Laplace force $F_{\mathrm{L}}$ and the surface tension $F_{\mathrm{s}}$. The Laplace force is caused by the pressure difference across the interface of a curved surface (the spherical water surface in Fig. 4a) and can be obtained through pressure difference multiplied by the wetting area as $F_{\mathrm{L}}$ $=-\kappa \gamma \cdot \pi R^{2}$ [6], where $\kappa$ is the mean curvature of the curved liquid surface with $\kappa=\left(1 / r_{1}\right)+\left(1 / r_{2}\right)$. Because the water droplet is assumed to be spherical and the nano-film wraps the water droplet completely, both the principal radii of curvature equal to the radius of water droplet $\left(r_{1}=r_{2}=R\right)$ and are independent of the contact angle $\theta_{1}$ and $\theta_{2}$, which is different from that of the meniscus between two rigid surfaces. The surface tension force acting on the nano-film is $F_{\mathrm{S}}=2 \pi R \gamma \cos \left(180^{\circ}-\theta_{1}\right)$ [6], which depends only on the contact angle $\theta_{1}$ between liquid and nano-film surface.

Hence, the total capillary force acting on the nano-film is

$F_{\mathrm{C}}=-\kappa \gamma \cdot \pi R^{2}+2 \pi R \gamma \cos \left(180^{\circ}-\theta_{1}\right)=-2 \pi R \gamma\left(1+\cos \theta_{1}\right)$

The van der Waals force acting in the domain outside the liquid area can be given as

$F_{\text {dry }}=\frac{A_{\text {dry }}}{6 \pi D_{0}^{3}}\left(b_{1} b_{2}-2 \pi R^{2}\right)$

where $D_{0}$ is the most intimately contact separation between the film and substrate. Therefore, the total adhesion force can be expressed as

$F_{\text {total }}=F_{\mathrm{C}}+F_{\mathrm{dry}}=-2 \pi R \gamma\left(1+\cos \theta_{1}\right)+\frac{A_{\mathrm{dry}}}{6 \pi D_{0}^{3}}\left(b_{1} b_{2}-2 \pi R^{2}\right)$

If the radius of liquid droplet is smaller than $R_{\mathrm{EC}}$, surface energy is not sufficient to render the thin film wrapping droplet completely. As a result, partial wrapping forms as shown in Fig. 4a. From the energy balance point of view, the following relation for the partial wrapping case can be found

$$
\begin{aligned}
\frac{D L}{2 R_{\mathrm{EC}}^{2}} & +\gamma_{\mathrm{SL}} L+\gamma\left(2 \pi R_{\mathrm{EC}}-L\right)=\frac{D L_{1}}{2 R^{2}}+\gamma_{\mathrm{SL}} L_{1}+\left(\gamma_{\mathrm{SV}}+\gamma\right) L_{2} \\
& +\gamma\left(2 \pi R-L_{1}-L_{2}\right)
\end{aligned}
$$

where $\gamma_{\mathrm{SL}}$ and $\gamma_{\mathrm{SV}}$ are the solid-liquid and solid-vapor interfacial tensions respectively, $L_{1}$ is the partial wrapping length and $L_{2}=L-L_{1}$ the detached length as the liquid radius $R$ is smaller $R_{\mathrm{EC}}$. Substituting $D L_{2} / 2 R_{\mathrm{EC}}^{2}=\gamma\left(1+\cos \theta_{1}\right) L_{2}$ and $L_{1}=2 R \phi$ into Eq. (9) yields

$\frac{D R \phi}{R_{\mathrm{EC}}^{2}}+2 \pi R_{\mathrm{EC}} \gamma=\frac{D \phi}{R}+2 \pi R \gamma$

Then the relationship between the radius of liquid droplet $R$ and the wetting angle $\phi$ can be founded as

$R=\frac{D \phi}{2 \pi \gamma R_{\mathrm{EC}}-\left(D \phi / R_{\mathrm{EC}}\right)}$

In this case, the capillary force also includes Laplace force and surface tension components,

$\begin{aligned} F_{C} & =-\kappa \gamma \cdot \pi x^{2}+2 \pi \gamma x \cos \left(\phi-\theta_{1}+90^{\circ}\right) \\ & =-2 \pi R \gamma \sin \phi\left[\sin \phi+\sin \left(\phi-\theta_{1}\right)\right]\end{aligned}$

where $x=R \sin \phi$ is the wetting radius.

Here, we assume that the convex area of the thin film equals to the surface area of the liquid droplet. The van der Waals force between the hemispherical thin film and substrate is negligible due 
(a)
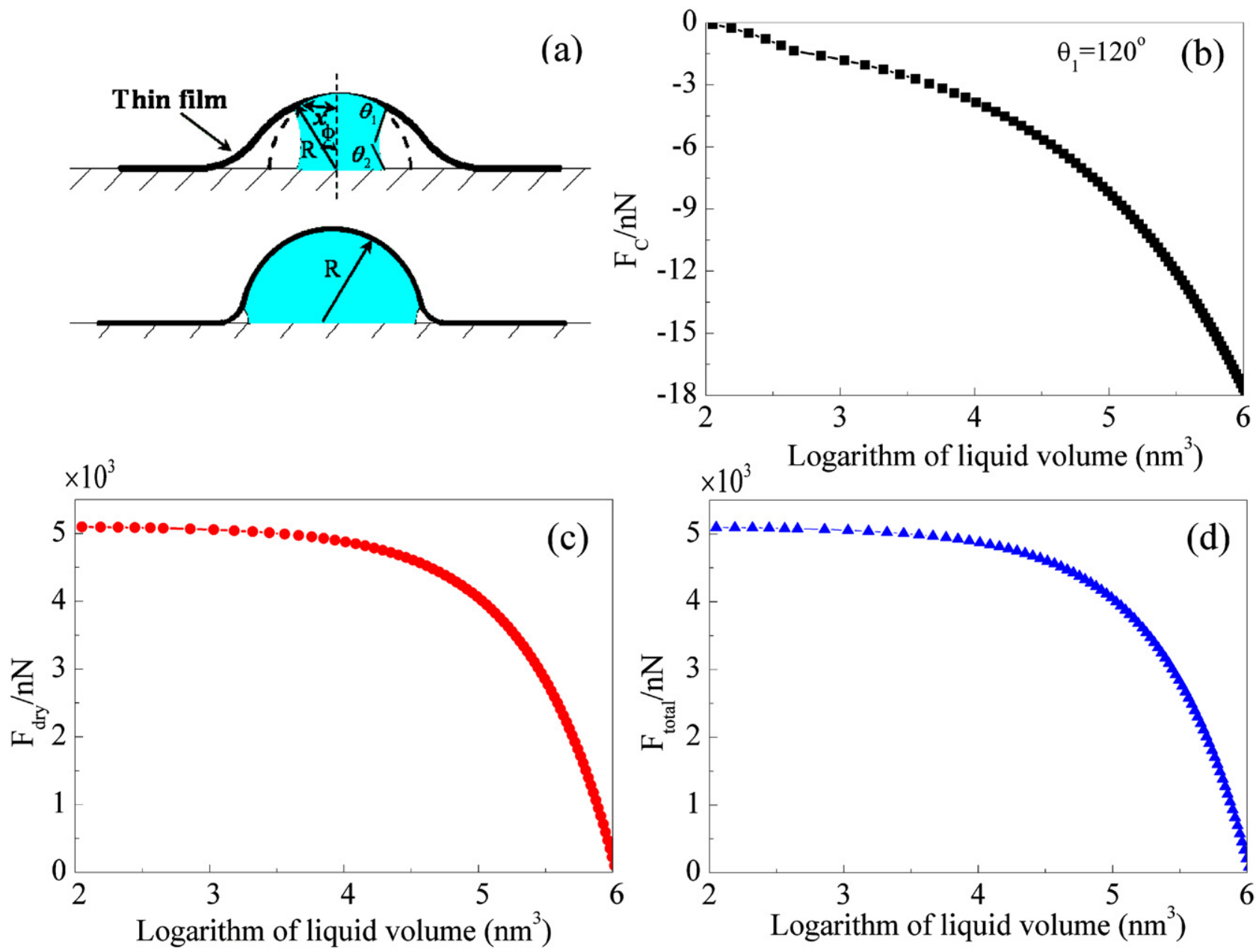

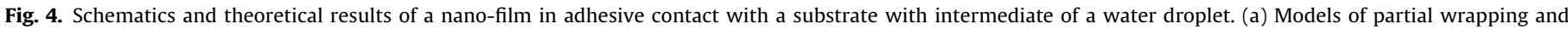

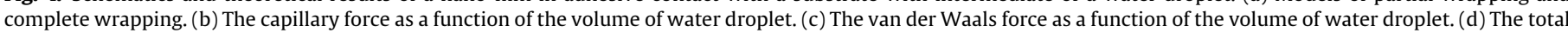
adhesion force as a function of the volume of water droplet.

to a large separation. Thus, the van der Waals force between the flat part of thin film and substrate can be expressed as the same as Eq. (7)

$F_{\mathrm{dry}}=\frac{A_{\mathrm{dry}}}{6 \pi D_{0}^{3}}\left(b_{1} b_{2}-2 \pi R^{2}\right)$

which yields the total adhesion force

$F_{\text {total }}=F_{C}+F_{\text {dry }}=-2 \pi R \gamma \sin \phi\left[\sin \phi+\sin \left(\phi-\theta_{1}\right)\right]$

$$
+\frac{A_{\mathrm{dry}}}{6 \pi D_{0}^{3}}\left(b_{1} b_{2}-2 \pi R^{2}\right)
$$

\section{Results and discussion}

Fig. 5 shows the total adhesion force as a function of the relative humidity with a set of values of $A_{\mathrm{dry}}=6.5 \times 10^{-20} \mathrm{~J}$, $A_{\text {wet }}=3.7 \times 10^{-20} \mathrm{~J}, A=6.5 \times 10^{-20} \mathrm{~J}$, and $b_{1}=b_{2}=200 \mathrm{~nm}$, which are similar to the parameters used by a spatula on geckos' feet. Because of a monolayer of water adsorbed on substrate and the most intimate separation between two surfaces, we take $D_{0}=h=0.3 \mathrm{~nm}$. From Fig. 5, one can see that the total adhesion force increases linearly with an increasing relative humidity, which qualitatively agrees with the experimental observations that the relative humid-

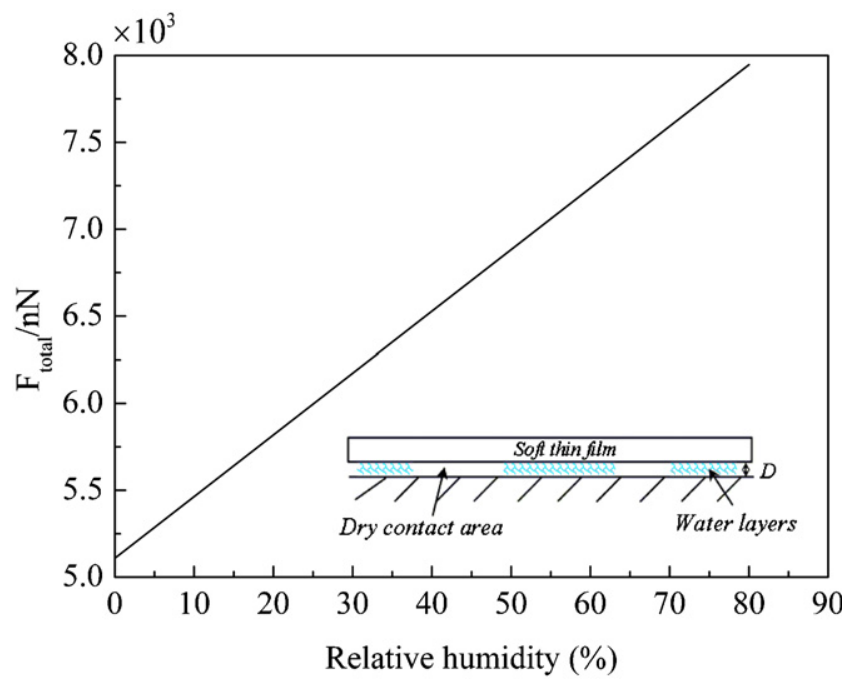

Fig. 5. The total adhesion force as a function of the relative humidity in the adhesion model of a nano-film in contact with a substrate. The intermediate is monolayer of water with a coverage ratio $\rho$. 
ity always enhances geckos' adhesion for both hydrophilic and hydrophobic surfaces [4].

One may note that, if the substrate has a rough surface and the effect of surface roughness is considered at different relative humidity, the pull-off force will be influenced by several factors, such as the height of the capillary bridge, the height of roughness peaks, the contact time and the wettability of the surface. Thus, different experimental phenomena could be found in different experiments due to different factors. For examples, in Delrio et al. [22], it is found that the adhesion energy is a constant and independent of relative humidity up to $60-70 \% R H$. However, in Van Zwol et al. [23], it is found that even for the lowest attainable relative humidity ( $\sim 2 \%$ ) very large capillary forces are still present. As for the experimental results found by Delrio et al. [22], Wang et al. [24] proposed a theoretical model based on a hierarchical rough surface topography to quantitatively explain the experimental observation and predicted a threshold relative humidity value. When the relative humidity is smaller than the critical value, there are no stable water bridges due to the surface roughness and adhesion is governed by dry adhesion, so that the adhesion energy almost keeps a constant. When the relative humidity is larger than the critical value, water menisci freely form and spread into some of the open gaps along the interface, then the water bridge is closed and its height is higher than the highest roughness peaks. Thus the adhesion energy will depend on the wetting area. This issue has also been pointed out by Van Zwol et al. [23]. However, the model in the present paper is about a smooth substrate and a monolayer of water molecules is assumed to adsorb on the smooth substrate at low relative humidity, which can be found in the experiment done by Huber et al. [4]. The water coverage increases with increasing relative humidity. At low relative humidity, the disjoining pressure can enhance the adhesion force as shown in Mate and Novotny [13].

Fig. 4(b-d) shows the capillary force, the van der Waals force and the total adhesion one as a function of the volume of droplet, respectively. The parameters used in Fig. 4(b-d) are taken according to the adhesion condition of geckos as $\theta_{1}=120^{\circ}$, $\gamma=0.072 \mathrm{~J} / \mathrm{m}^{2}, A_{\text {dry }}=6.5 \times 10^{-20} \mathrm{~J}, E=2 \mathrm{GPa}, \nu=0.3, D_{0}=0.3 \mathrm{~nm}$, and $b_{1}=b_{2}=200 \mathrm{~nm}$. It is found that the capillary force is a repulsive one and the absolute value increases with an increasing volume of droplet. The attractive van der Waals force decreases with an increasing volume of droplet. Both the capillary force and the van der Waals one lead to a decreasing total adhesion force when the volume of water droplet increases. As a simple way to illustrate the rationality of the above results, a live gecko was allowed to climb a "dry" vertical surface. Once sprayed with water, the gecko was unable to adhere to the surface. This technique of spraying geckos with water to remove them from vertical surfaces is well known to zoologists [5]. Large volume of water droplet reduces, even vanishes the total adhesion force of geckos.

\section{Conclusion}

The adhesion of a bio-inspired nano-film in contact with a substrate with intermediate water layer or water droplet is investigated theoretically. It is found that the disjoining pressure between water molecules and solid surface compensates the reduction of van der Waals force and leads to an enhancing adhesion. As the relative humidity is larger than $90 \%$, water layer transforms to water droplet. The capillary force is a repulsive one. Van der Waals force and the total adhesion one decrease with an increasing volume of water droplet. The results for the two different stages are consistent well with the experimental observations by Huber et al. [4] and the common sense [5], respectively, which may show significant insights into the fabrication of dry adhesives and robotic systems inspired by the gecko's adhesion mechanism.

\section{Acknowledgements}

The work reported here is supported by NSFC through Grants $\# 10972220$, \#10732050, and \#11021262. In addition, the authors should give their many thanks to the two anonymous reviewers for their helpful and professional suggestions.

\section{References}

[1] K. Autumn, Y.A. Liang, S.T. Hsieh, W. Zesch, W.P. Chan, T.W. Kenny, R. Fearing, R.J. Full, Nature 405 (2000) 681-685.

[2] G. Huber, S.N. Gorb, R. Spolenak, E. Arzt, Biol. Lett. 1 (2005) 2-4.

[3] K. Autumn, M. Sitti, Y.C.A. Liang, A.M. Peattie, W.R. Hansen, S. Sponberg, T.W. Kenny, R. Fearing, J.N. Israelachvili, R.J. Full, Proc. Natl. Acad. Sci. U.S.A. 99 (2002) 12252-12256.

[4] G. Huber, H. Mantz, R. Spolenak, K. Mecke, K. Jacobs, S.N. Gorb, E. Arzt, Proc. Natl. Acad. Sci. U.S.A. 102 (2005) 16293-16296.

[5] W.X. Sun, P. Neuzil, T.S. Kustandi, S. Oh, V.D. Samper, Biophys. J. 89 (2005) L14-L17.

[6] T.W. Kim, B. Bhushan, J. R. Soc. Interface 5 (2008) 319-327.

[7] F.M. Orr, L.E. Scriven, A.P. Rivas, J. Fluid Mech. 67 (1975) 723-742.

[8] M. Prowse, M. Wilkinson, J. Puthoff, G. Mayer, K. Autumn, Acta Biomater. 7 (2011) 733-738.

[9] J.B. Puthoff, M.S. Prowse, M. Wilkinson, K. Autumn, J. Exp. Biol. 213 (2010) 3699-3704.

[10] B. Chen, H. Gao, Int. J. Appl. Mech. 2 (2010) 1-9.

[11] F.W. DelRio, M.L. Dunn, M.P. de Boer, Scr. Mater. 59 (2008) 916-920.

[12] B.V. Derjaguin, N.V. Churaev, J. Colloid Interf. Sci. 49 (1974) 249-255.

[13] C.M. Mate, V.J. Novotny, J. Chem. Phys. 94 (1991) 8420-8427.

[14] C.M. Mate, J. Appl. Phys. 72 (1992) 3084-3090.

[15] N.V. Churaev, Colloid Polym. Sci. 253 (1975) 120-126.

[16] J. Israelachvili, Intermolecular and Surface Forces, Academic Press, London, 1991.

[17] S. Basu, M.M. Sharma, J. Colloid Interf. Sci. 181 (1996) 443-455.

[18] B. Dai, L.G. Leal, A. Redondo, Phys. Rev. E 78 (2008) 061602.

[19] L.R. White, J. Colloid Interf. Sci. 343 (2010) 338-343.

[20] C. Py, P. Reverdy, L. Doppler, J. Bico, B. Roman, C.N. Baroud, Eur. Phys. J. Special Top. 166 (2009) 67-71.

[21] J. Bico, B. Roman, L. Moulin, A. Boudaoud, Nature 432 (2004) 690.

[22] F.W. DelRio, M.L. Dunn, L.M. Phinney, C.J. Bourdon, M.P. De Boer, Appl. Phys. Lett. 90 (2007) 163104.

[23] P.J. van Zwol, G. Palasantzas, J.T.M. De Hosson, Phys. Rev. E 78 (2008) 031606.

[24] J.Z. Wang, J. Qian, H.J. Gao, Langmuir 25 (2009) 11727-11731. 